\title{
Syncope as a Long Term Effect of Coronavirus ("Long Covid") in an Afro Caribbean Patient: A Case Report
}

\section{Felix Nunura ${ }^{*}$, Edwin Tulloch-Reid ${ }^{2}$, Ahmed Soliman ${ }^{2}$, Safiya Weekes ${ }^{3}$, Eldhose Basil $^{3}$, Daina Baugh ${ }^{3}$ and Ernest Madu ${ }^{1}$}

${ }^{1}$ Department of Cardiovascular Medicine, Heart Institute of the Caribbean, Kingston, Jamaica

${ }^{2}$ Department of Interventional Cardiology, Heart Institute of the Caribbean, Kingston, Jamaica

${ }^{3}$ Department of Medicine, Heart Institute of the Caribbean, Kingston, Jamaica

*Corresponding Author: Felix Nunura, Department of Cardiovascular Medicine, Heart Institute of the Caribbean, Kingston, Jamaica.
Received: May 31, 2021

Published: July 28, 2021

(C) All rights are reserved by Felix Nunura., et al.

\begin{abstract} Vagal Syncope.

Keywords: COVID-19; SARS-CoV-2; Autonomic Nervous System
\end{abstract}

While most persons with COVID-19 recover and return to normal health, some patients can have symptoms that can last for weeks or even months after recovery from acute illness. We describe an Afro Caribbean patient with symptoms of 'long COVID', and we posit that this condition may be related to a virus- or immune-mediated disruption of the autonomic nervous system resulting in a Vaso

\section{Introduction}

The SARS-CoV-2 (COVID-19) pandemic has caused unprecedented morbidity, mortality and disruption across the world. Following the initial phase, healthcare services are focusing now on controlling new cases and planning rehabilitation strategies. 'Postacute COVID [1] refers to persistent symptoms 3 weeks after COVID-19 infection, while 'Chronic COVID' describes symptoms lasting more than 12 weeks [2]. Several months on from the declaration of the COVID-19 pandemic, new symptom patterns and syndromes such as 'long COVID' are emerging. Some of these patterns may be explained by autonomic instability and may result from deconditioning, hypovolemia or immune- or virus-mediated neuropathy. Orthostatic intolerance (OI) syndromes include orthostatic hypotension $(\mathrm{OH})$, vasovagal syncope (VVS) and postural orthostatic tachycardia (POTS) have started being reported [3]. We report the case of a 50-year-old Afro Caribbean man with multiple comorbidi- ties and recurrent episodes of extreme fatigue, headache, recurrent chest pain, dizziness, Blood Pressure instability, general weakness after standing and finally three episodes of Syncope after 16 weeks of having a Positive Test for Covid-19. Clinicians must be aware that prompt and correct diagnosis of this autonomic dysfunction with careful management are essential for recovery.

\section{Case Presentation}

A 50-year-old Afro Caribbean male with past medical history of hypertension, hyperlipidemia, obesity, sleep disorder, cervical spondylosis, and 1 vessel coronary artery disease treated with PCI (June 2020). His regular therapy included Losartan $100 \mathrm{mg}$ O.D., Nebivolol 5 mg O.D., Clopidogrel 75 mg O.D., Atorvastatin 40 mg O.D. On August 2020 he tested positive for COVID-19 and he showed minimal viral infection symptoms so he was asked to selfquarantine and manage his symptoms at home. Approximately 16 
weeks after recovery (January $12^{\text {th }}, 2021$ ) he presented to our HIC outpatient clinic for a follow-up visit reporting recent recurrent (three) syncopal episodes for 1 week. The syncopal episodes were preceded by a prodromal of light-headedness and tunnel vision. These were witnessed and lasted 10 seconds to 1 minute and usually occurred after standing for short periods of time (less than 5 minutes). He also has been complaining of severe headaches, dizziness, low blood pressure, frequent dehydration, extreme fatigue and general weakness on standing. Of note, at one point, our patient could not stand for more than 5-10 minutes because of dizziness and a sense of weakness. When seen at in our office his BP was mildly elevated at 154/84 mmHg and $\mathrm{HR}$ of $82 \mathrm{bpm}$ was recorded, otherwise his general Physical Exam was unremarkable. His ECG showed Normal sinus Rhythm and no evidence of acute ischemia. ectopy or arrhythmias The Echocardiogram showed normal bi-ventricular systolic function with an Ejection Fraction of 65\%, Grade 1(mild) diastolic dysfunction but no significant structure cardiac abnormalities. A Stress Echocardiogram was done which was negative for exercise -induced ischemia or LV dilatation. A 24hr Holter ECG was negative for arrhythmias. His Ambulatory Blood Pressure Monitoring (24 hours showed a 24 hours average BP of 164.0/97.3 $\mathrm{mmHg}$. Laboratory investigations showed a mildly elevated serum creatinine of 129 umol/L (Normal range 64 - 111 umol/L) but normal complete blood count, cardiac enzymes, electrolytes, lipid profile. A CT Brain Scan was reported initially normal.

\section{Head-up tilt table (HUTT) testing (Figure 1)}

The patient did not need sublingual nitrate as he met criteria for positive Test within 29 minutes of upright Tilt. The Maximum recorded BP was 169/95 $\mathrm{mmHg}$ at minute 1 , and the lowest recorded BP was $88 / 63 \mathrm{mmHg}$ occurring at: $29^{\text {th }} \mathrm{min}$ of testing. Regarding the Heart rate monitoring recorded values, the maximum recorded rate was $90 \mathrm{bpm}$ occurring at: $16^{\text {th }}$ min of testing and the lowest recorded rate was $73 \mathrm{bpm}$ (a $27 \mathrm{bpm}$ difference) occurring at: $29^{\text {th }}$ min of testing. Tilt Testing response: as seen in the figure 1, the HUTT test showed a blood pressure of $155 / 98 \mathrm{mmHg}$ and a pulse rate of 80 beats/min at baseline while in the supine position. His blood pressure remained stable until the minute $10^{\text {th }}$ when it began to decrease progressively for the next 10 minutes (PreSyncopal Phase). A Pre syncopal increase in Diastolic Blood Pressure and decrease in Pressure Pulse were also noted and finally his Blood Pressure fell to $88 / 63 \mathrm{mmHg}$ at minute $29^{\text {th }}$ into head upright tilt. His heart rate however after an initial decrease to $78 \mathrm{bpm}$ at minute $4^{\text {th }}$ it slowly increased gradually to $90 \mathrm{bpm}$ (peak HR) at 16 minutes and also at 23 minutes respectively and then showed a progressive decline to its lowest value of $65 \mathrm{bpm}$ immediately after he experienced transitory loss of consciousness (TLOC) which was preceded by symptoms of dizziness, tunnel vision and general weakness. The HUTT Testing response was reported as positive for VVS (reflex syncope, neutrally mediated hypotension) of mixed type but predominantly vasodepressor Type (BP fell to $88 \mathrm{mmHg}$ ) but associated with a $10 \%$ drop in the Heart Rate at the time of syncope.(from the peak of $90 \mathrm{bpm}$ to $73 \mathrm{bpm}$ ) but it never fall to less than $40 \mathrm{bpm}$ or declining to complete asystole. Notably his heart rate continued to falling immediately after Syncope to a nadir of $65 \mathrm{bpm}$ in the $31^{\text {st }}$ minute.

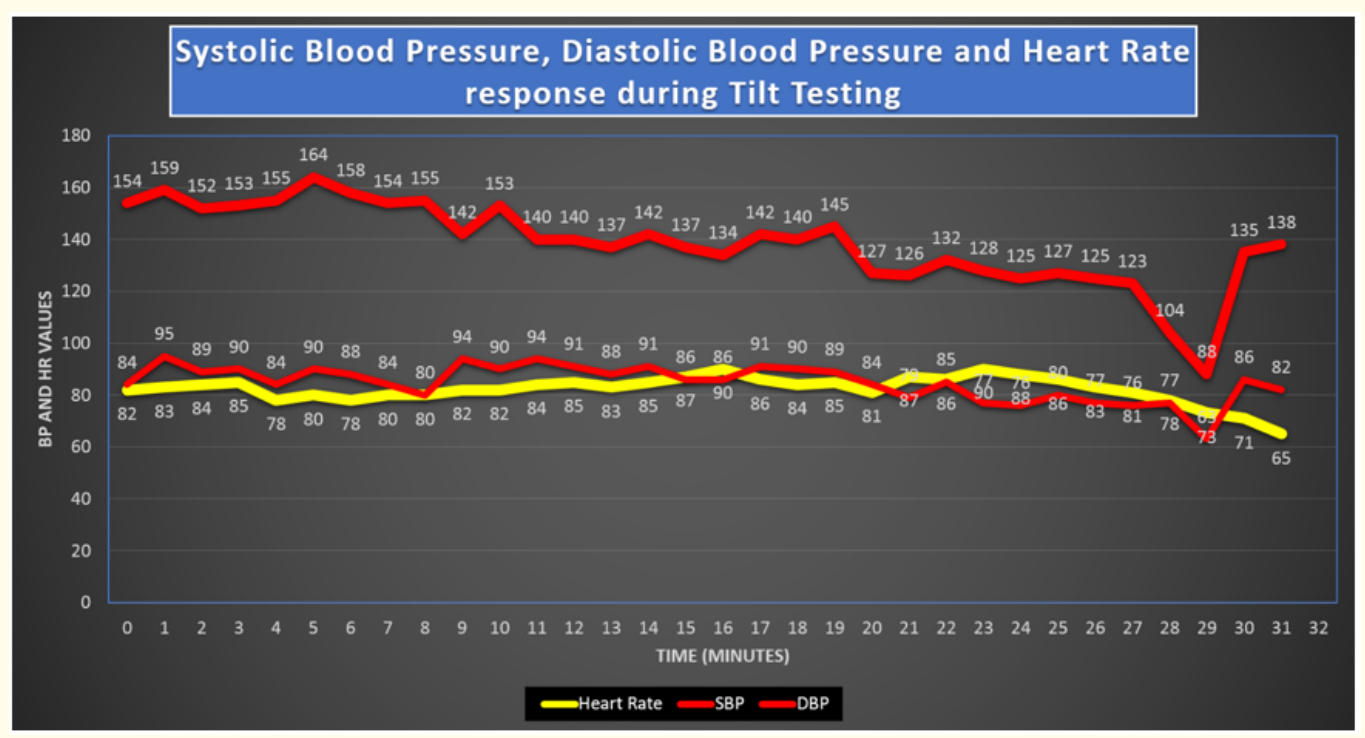

Figure 1: Continuous heart rate (yellow line) and blood pressure (red line) monitoring during a head-up tilt table test from an afro caribbean individual with long COVID and orthostatic intolerance (recurrent VVS) following COVID-19 infection in August 2020. 


\section{Treatment and evolution}

The patient was recommended that he should maintain a high fluid and salt intake and his antihypertensives were modified. A moderate improvement in his orthostatic symptoms was experienced within two weeks he and was able to resume performing most of his normal activities of daily living. He denied any further episodes of presyncope or syncope and showed clear improvement in his original symptoms of light-headedness, dizziness, fatigue). His blood pressure remained relatively normalized. He however continued to complain of severe headaches although these were less severe than at the outset.

\section{Discussion}

We report a 50 years-old afro Caribbean male patient with multiple co morbidities who approximately 16 weeks after post COVID-19 infection began experiencing fatigue, orthostatic dizziness, recurrent chest pain, lightheadedness, severe headache, pre syncope and finally three consecutive Syncope episodes and we posit that this clinical symptoms might be consistent with Autonomic Dysfunction associated with a Long COVID syndrome ("Chronic COVID" in this case). The exact mechanism of post-COVID-19 Orthostatic Intolerance syndromes (VVS in this case) is unknown and is suspected to be an autoimmune as well as an autonomic nervous system disorder. It has been hypothesized that COVID-19 infection affects the autonomic nervous system [4]. The relationship between autoimmunity provoked and autonomic dysfunction is complex: the well-documented cytokine response storm of COVID-19 [5] results from sympathetic activation inducing proinflammatory cytokine release [6,7] Conversely, vagal stimulation results in an anti-inflammatory responses [5], suggesting possible therapeutic targets in the autonomic nervous system. Alternatively, COVID-19 related autonomic dysfunction could be mediated by the virus itself. Immune-mediated neurological syndromes have been described [8] It is also well established that autonomic disorders such as $\mathrm{OH}$ and POTS are associated with autoantibodies [9], for example to alpha or beta adrenoceptors and muscarinic receptors [10-12]. Cohort studies describe commonly preceding infections in POTS [13] as well as a link with autoimmune biomarkers and autoimmune disorders [14]. We speculate that might be an underlying autoimmune component to the post-COVID syndrome that we are reporting here. During the HUTT Testing our patient showed a response of marked hypotension (vasodepressor response) and inadequate slow heart rate (cardio inhibition response) that lead to the TLOC. The pathophysiology links on an abnormal autonomic response to orthostasis (standing up/upright tilt). It is known that when a healthy person stands, blood pools in the pelvis and legs, reducing venous return to the heart. This is detected by barore- ceptors in the heart and aorta, which respond by increasing sympathetic neural and adrenergic tone (mediated by norepinephrine and epinephrine respectively). This results in tachycardia (thus compensating for reduced stroke volume) which in turn triggers 'compensatory' vasoconstriction in the splanchnic vascular bed leading to increased venous return to the heart. On the other hand in orthostatic intolerance (OI) the exaggerated release of epinephrine and norepinephrine causes pronounced tachycardia, which is experienced as palpitations, breathlessness and chest pain ( which are also common symptoms of 'long COVID'). The very high catecholamine levels attained can also lead to paradoxical vasodilatation, sympathetic activity withdrawal and vagal activation resulting in hypotension, dizziness, sometimes bradycardia and ultimately syncope [15-19]. In addition Postural orthostatic tachycardia syndrome (POTS) the most prevalent chronic cardiovascular dysautonomia among young and middle-age individuals, predominantly women, characterized by chronic orthostatic intolerance, abnormal heart rate (HR) increase on standing, and deconditioning has been postulated to have post-viral autoimmune activation as a possible etiology [20] and recently a Long-Haul Post-COVID-19 Symptoms Presenting as a Variant of Postural Orthostatic Tachycardia Syndrome (POTS) has been reported for the Swedish researchers [21] as major clinical centers in Sweden have witnessed an inflow of patients with chronic symptoms (Postural lightheadedness/ vertigo, palpitations, dyspnea and fatigue) following initial outpatient care for coronavirus disease-2019 (COVID-19) infection, suggestive of postural orthostatic tachycardia syndrome. Thus, until proved to the contrary we believe that our patient had an OI syndrome (recurrent VVS) as an unfortunate clinical manifestation of "Long COVID".

\section{Conclusion}

In the acute phase, coronavirus disease-2019 (COVID-19) causes multiple complications including pneumonia, respiratory distress syndrome, liver injury, cardiac injury, and prothrombotic coagulopathy. Although long-term consequences remain unknown [22] the rationale, physiology and management strategies for Autonomic dysfunction in 'long COVID' have begun to be considered in the medical literature [23]. It is currently clear that even several weeks or months of an acute infection caused by severe acute respiratory syndrome coronavirus 2 (SARS-CoV-2) new symptom patterns and syndromes which constitute the sub-acute or chronic 'LONG COVID' are emerging and that some of these clinical sceneries may be associated by secondary autonomic disorders. The basis for these is probably multi factorial resulting from deconditioning, hypovolemia and possible immune- or virus-mediated neuropathy. As a clinicians we must be aware that promptly establishing the 
correct diagnosis of possible "Autonomic Dysfunction post COVID" is essential for the improvement in quality of life for these patients.

\section{Funding Support and Author Disclosures}

The authors report that we have no relationships relevant to the contents of this paper to disclose.

\section{Bibliography}

1. Greenhalgh T., et al. "Management of post-acute Covid-19 in primary care". BMJ 370 (2020): m3026.

2. Chan AT., et al. "The Coronavirus Pandemic Epidemiology (COPE) Consortium: A call to action". Cancer Epidemiology, Biomarkers and Prevention 29 (2020): 1283-1289.

3. Khail Kanjwal., et al. "New-onset Postural Orthostatic Tachycardia Syndrome Following Coronavirus Disease 2019 Infection". Innovations in Cardiac Rhythm Management 11.11 (2020): 4302-4304.

4. Goldstein DS. "The extended autonomic system, dyshomeostasis, and COVID-19". Clinical Autonomic Research 30 (2020): 299-315.

5. Fudim M., et al. "Implications for neuromodulation therapy to control inflammation and related organ dysfunction in COVID-19". Journal of Cardiovascular Translational Research 13 (2020): 894-899.

6. Konig MF., et al. "Preventing cytokine storm syndrome in COVID-19 using alfa-1 adrenergic receptor antagonists". Journal of Clinical Investigation 137 (2020): 3345-3347.

7. Staedtke V., et al. "Disruption of a self-amplifying catecholamine loop reduced cytokine release syndrome". Nature 564 (2018): 273-277.

8. Guilmot A., et al. "Immune-mediated neurological syndromes in SARS-CoV-2-infected patients". Journal of Neurology (2020).

9. Ruzieh M., et al. "The role of autoantibodies in the syndromes of orthostatic intolerance: a systematic review". Scandinavian Cardiovascular Journal 51 (2017): 243-247.

10. Li H., et al. "Agonistic autoantibodies as vasodilators in orthostatic hypotension: a new mechanism". Hypertension 59 (2012): 402-408.
11. Fedorowski A., et al. "Antiadrenergic autoimmunity in postural tachycardia syndrome”. Europace 19 (2017): 1211-1219.

12. Li H., et al. "Autoimmune basis for postural tachycardia syndrome". Journal of the American Heart Association 3 (2014): e000755.

13. Watari M., et al. "Autoimmune postural orthostatic tachycardia syndrome". Annals of Clinical and Translational Neurology 5 (2018): 486-492.

14. Blitshteyn S., et al. "Autonomic dysfunction and HPV immunization: an overview". Immunology Research 66 (2018): 744754.

15. Gross A. "Fatigue plagues thousands suffering post-coronavirus symptoms". Financial Times, 3 August (2020).

16. Freeman R., et al. "Orthostatic hypotension: JACC State-of-theArt Review". Journal of the American College of Cardiology 72 (2018): 1294-1309.

17. Jardine DL., et al. "The pathophysiology of the vasovagal response". Heart Rhythm 15 (2018): 921-929.

18. Fenton AM., et al. "Vasovagal syncope". Annals of Internal Medicine 133 (2000): 714-725.

19. Fedorowski A. "Postural orthostatic tachycardia syndrome: clinical presentation, aetiology and management". Journal of Internal Medicine 285.4 (2019): 352-366.

20. Vernino S and Stiles LE. "Autoimmunity in postural orthostatic tachycardia syndrome: current understanding". Autonomic Neuroscience 215 (2018): 78-82.

21. Johansson M., et al. "Long-haul post-COVID-19 symptoms presenting as a variant of postural orthostatic tachycardia syndrome: The Swedish experience". JACC Case Report 3.4 (2021): 573-580.

22. Wiersinga WJ., et al. "Pathophysiology, transmission, diagnosis, and treatment of coronavirus disease 2019 (COVID-19): a review". JAMA 324 (2020): 782-793.

23. Dani Melani., et al. "Autonomic Dysfunction in "long COVID": rationale, physiology and management strategies". Clinical Medicine 21.1 (2020).

Volume 5 Issue 8 August 2021

(C) All rights are reserved by Nunura Felix., et al. 